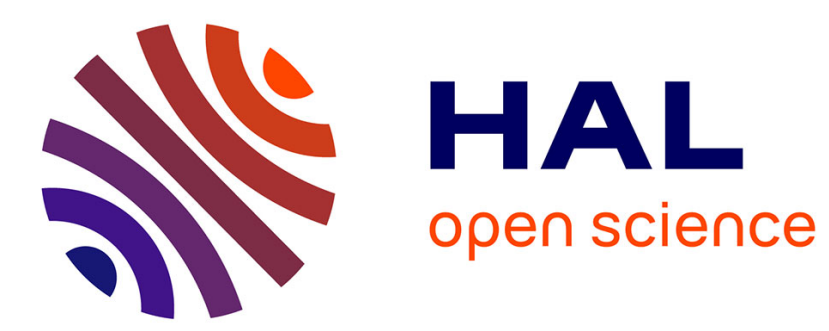

\title{
Diderot dans le Journal encyclopédique de P. Rousseau (1756-1770) : la puissance d'un modèle Jacques Wagner
}

\section{To cite this version:}

Jacques Wagner. Diderot dans le Journal encyclopédique de P. Rousseau (1756-1770) : la puissance d'un modèle. Recherches sur Diderot et sur l'Encyclopédie, 2021. hal-03244525

HAL Id: hal-03244525

https://hal.uca.fr/hal-03244525

Submitted on 1 Jun 2021

HAL is a multi-disciplinary open access archive for the deposit and dissemination of scientific research documents, whether they are published or not. The documents may come from teaching and research institutions in France or abroad, or from public or private research centers.
L'archive ouverte pluridisciplinaire HAL, est destinée au dépôt et à la diffusion de documents scientifiques de niveau recherche, publiés ou non, émanant des établissements d'enseignement et de recherche français ou étrangers, des laboratoires publics ou privés. 


\section{Diderot dans le Journal encyclopédique de P. Rousseau (1756-1770) : la puissance d'un modèle}

En choisissant de donner le titre de Journal Encyclopédique au périodique qu'il décida de lancer en 1755, P. Rousseau se classait, d'une part, parmi les hommes de lettres favorables aux Philosophes modernes et à Diderot surtout, ce qui lui vaudrait l'inimitié immédiate des partisans de la tradition "; d'autre part, il lui faudrait concilier le régime à temporalité courte d'un périodique avec le temps long de la réflexion plus adapté à l'exercice encyclopédique. D'autres titres étaient à la disposition de P. Rousseau soucieux d'en chercher un de qualité pour le sien, et entre autres celui de Journal littéraire qui avait disparu dès 1732 sans succession revendiquée. II pouvait même adopter une variante : Journal de littérature française et étrangère qui fut tentée en 1785 , comme suite de la Gazette de Littérature $(1770)^{2}$. Tout cela, impliquant moins d'exigences, aurait facilité son travail journalistique en l'autorisant à se maintenir dans le sillon des compilations habituelles, mais aurait manqué de panache. Or il avait l'ambition élevée et convoqua 'tout le monde savant' de l'Europe, et « les Corps le plus célèbres, les Compagnies les plus florissantes, les Artistes les plus fameux, à contribuer au succès de cette entreprise ${ }^{3}$, avec l'idée de constituer, autour de lui, une "société de gens de lettres », la plus large possible, à la façon de Diderot ${ }^{4}$. Pour acquérir l'aura 'encyclopédique', il fallait en accepter le prix : tempêtes judiciaires et politiques ${ }^{5}$, ce qui lui assurait d'être adoubé par les victimes des censures religieuses ou parlementaires. II s'engagea, en outre, à protéger les savants des accusations proférées par « les hommes qui les regardent comme impies et sacrilèges » (JE 15 nov. 1756, p.17). Pour être digne de ceux qu'il nomme les « Grands Hommes », il rattacha, par un récit flatteur, la naissance de son projet à Bacon et à l'Encyclopédie ${ }^{6}$. II promit beaucoup et eut raison de signaler que « l'étendue de (ses) promesses a fait douter de la possibilité de les remplir » (JE, 1er janvier, 1756, Avertissement, p. 11).

II se lança toutefois dans l'aventure et, en dépit des difficultés, en sortit grandi et ne disparut qu'avec la monarchie en 1793, parcourant un demi-siècle de vie culturelle sans défaillir : "L'incertitude (de réussir), comme il l'assure lui-même, avec l'énergie optimiste de son époque, a redoublé notre zèle » (ibid). Le zèle était-il pour autant un gage suffisant de réussite ? Accordait-il au désir d'un rapprochement avec le champ encyclopédique la légitimité d'un intervenant autorisé et reconnu ? Le titre ne cachait-il pas une vantardise

\footnotetext{
${ }^{1}$ II ne pouvait ignorer le sort que Fréron avait réservé aux Pensées sur l'Interprétation de la nature de Diderot, dans le premier compte rendu de son nouveau périodique L'Année littéraire en 1754 au ton particulièrement virulent (A.L. tome I, lettre 1, p.1-14)

${ }^{2}$ II avait un instant envisagé de dénommer son périodique, Journal étranger, choix refusé par Malesherbes.

${ }^{3}$ Avertissement des associés à ce journal (1er janvier 1756, p. iv).

${ }^{4} \mathrm{P}$. Rousseau a bien retenu les arguments de Diderot explicitant le choix d'une pluralité de collaborateurs : "Un Dictionnaire universel et raisonné des Sciences et des Arts ne peut être l'ouvrage d'un homme seul. Je dis plus ; je ne crois pas que ce puisse être l'ouvrage d'aucune des sociétés littéraires ou savantes qui subsistent » (Enc., vol.V, p.635va)

5 Outre les pamphlets divers, les protestations du clergé liégeois dès 1757 , la condamnation de 1759 par les Théologiens de Louvain, l'interdiction de maintenir son siège à Liège et son transfert à Bouillon, le JE eut à souffrir de la cessation du soutien important du Mercure de France journal de la Cour et des mondains : ce dernier mit fin, en 1760, à ses annonces favorables au Journal, insérées à partir de novembre 1758 par son directeur d'alors, Marmontel comme une promesse officieuse de protection par le gouvernement français ; entré au Mercure le 27 avril 1758, Marmontel fut démis de sa fonction en janvier 1760 et le JE perdit sa protection. Il ne la retrouva qu'en juillet 1769 .

${ }^{6} J E$, 1 er janvier 1756, p.XII. Le littérateur impartial fut d'un avis différent, déclarant que « le plan du Journal Encyclopédique était calqué sur celui du Journal Étranger » (Tome 1,1760, p. 213).
} 
commerciale ? Les rédacteurs de la Bibliographie parisienne se sentirent un jour obligés d'inclure des 'extraits' dans leurs annonces des publications récentes pour contrecarrer le pouvoir souvent fallacieux des titres : "Le titre d'un ouvrage est communément très séduisant. Mais l'ouvrage y répond-il toujours ? Combien de fois n'a-t-on pas été exposé au regret de s'en être procuré la possession sur ce seul indice ? $\gg^{7}$. N'en allait-il pas de même dans le cas des périodiques qui promettaient plus qu'ils n'offraient, surtout dans le cas aussi étonnant d'un Journal qui se prétendit encyclopédique, par l'esprit et par la quantité ${ }^{8}$. L'enjeu était d'autant plus fort que le qualificatif inclus dans son titre avait alors acquis un prestige considérable. Le sérieux et le succès que, depuis 1750 , ce terme conférait à une entreprise littéraire, grâce au Prospectus de novembre 1750, puis grâce, en 1751, au long discours préliminaire de d'Alembert inséré dans le volume I du Dictionnaire et grâce, enfin en 1755, au vaste article de Diderot intitulé « Encyclopédie » (Vol.5), ne pouvaient qu'avoir marqué les esprits, sans compter le bruit des interdictions du Conseil d'État, des attaques du P. Geoffroy (Nouvelles ecclésiastiques) dès 1752, puis de Fréron en 1754 (L'Année littéraire). Le qualificatif apparaissait donc chargé d'un poids, d'une réputation et d'un attrait qui ne pouvaient échapper à $P$. Rousseau, du même âge que Diderot. Il lui fallut donc renouveler la conception du journalisme littéraire et « découvrir de nouvelles ressources » (JE, 1er janvier 1756, p. XI). Sous l'ombre magnétique de son modèle encyclopédique, il chercha à combler le déficit d'image et de sérieux qui séparait son entreprise périodique de la haute figure de sa référence inaugurale. II connaissait le mépris avec lequel Diderot parlait de la presse périodique et inséra des phrases entières de son modèle dans son Avertissement introductif, repérées lors de sa lecture de l'article « Encyclopédie », sans avouer sa source : il en partageait trop bien les raisons, comme il le déclara en plagiant Diderot :

Quant aux journaux,...resserrés dans le cercle étroit du temps présent, ils n'osent franchir cet espace déterminé ; d'ailleurs, on leur impute ${ }^{9}$ de n'être qu'une histoire momentanée des ouvrages et des auteurs, où l'on rend compte indistinctement des efforts heureux ou malheureux ${ }^{10}$ et où conséquemment pour un feuillet qui mérite de l'attention, on traite au long une infinité de volumes qui tombent dans l'oubli avant que le dernier journal de l'année ait paru. Ainsi loin d'être destinés à l'instruction générale et permanente de l'espèce humaine, ils le sont tout au plus à la satisfaction passagère de la curiosité publique $»^{11}(J E, 15$ novembre 1756, p.7).

\footnotetext{
7 'Avis des rédacteurs' de la Bibliographie parisienne, juillet 1771, p.viii.

${ }^{8}$ Pour les amateurs d'études statistiques menées sous l'égide des méthodes quantitatives de l'histoire sérielle des Annales vers 1970, voir notre thèse Lecture et société dans le Journal encyclopédique de $P$. Rousseau (1756-1785), Clermont-Ferrand, 1987.

${ }^{9}$ Cette imputation vise discrètement Diderot qui décrivit brutalement et sans ménagement, à son habitude, les défauts structurels des périodiques: " II y a bien de la différence entre une Encyclopédie et une collection de journaux...". Dans notre citation, les phrases soulignées sont recopiées littéralement de l'article de Diderot («Encyclopédie », p.645va).

10 Sur cette indistinction désolante des ouvrages à analyser, $P$. Rousseau ne fait-il pas écho à Diderot qui réclamant la révélation du moyen des expériences physiques, apportait une nuance de taille : «Au reste, quand je demande la révélation du moyen, j'entends de celui par lequel on a réussi : on ne peut être trop succinct sur ceux qui n'ont point eu de succès " (De l'interprétation de la nature, XXXIX, éd. Vernière, Classiques Garnier, 1961, p. 215. Le choix sera pour P. Rousseau un conseil de poids.

${ }^{11}$ La dernière phrase de cette) citation est également recopiée de Diderot («Encyclopédie ») avec un remaniement adoucissant le discours. $P$. Rousseau avait repéré que Diderot avait déjà distingué une pédagogie restreinte, propre à ne satisfaire qu'un goût superficiel à la mode par la seule quantité, d'une pédagogie efficace : "Nos faiseurs de cours d'expériences ressemblent un peu à celui qui pense avoir donné un grand repas parce qu'il aurait eu beaucoup de monde à table. II faudrait donc s'attacher principalement à irriter l'appétit, afin que plusieurs, emportés par le désir de la satisfaire, passassent de la
} 
Diderot s'appesantissait sur cette faiblesse du travail périodique, le vouant à l'inutilité et à la vanité : «Mais que devient l'extrait quand le livre est oublié ? », alors que l'Encyclopédie serait immortelle : «Un dictionnaire universel et raisonné est destiné à l'instruction générale et permanente de l'espèce humaine ». II concluait sur une clausule assassine : « Ils sont peu lus des gens de lettres » (art. « Encyclopédie », vol. V,1755, p. 645)

Toutefois, le Journal ne se défaussa pas : il ouvrit abondamment ses pages à Diderot et à l'Encyclopédie. Entre 1756 et 1770, il rendra compte fidèlement, entre 1756 et 1770, des douze volumes de l'Encyclopédie, parus entre 1755 (vol.V) et 1765 (vol.XVII), à raison d'un compte rendu par numéro, sans négliger les articles dits 'omis' inclus dans ce dernier volume soit un total de 96 comptes rendus portant souvent sur plusieurs articles du Dictionnaire (de deux à quatre) ; et sans oublier les Suppléments qu'il présente de 1777 à 1779 (jusqu'à l'article «Vers », janvier, p. 94) en 35 comptes rendus ${ }^{12}$. Nous avons pu montrer qu'à partir de 1758, les articles de l'Encyclopédie remplaçaient progressivement un grand nombre de comptes rendus consacrés habituellement aux catégories bibliographiques des Sciences et Arts et de l'Histoire ${ }^{13}$ et laissaient dans l'ombre les livres de Religion qui ne retrouveront grâce qu'en 1759, lors de la disparition des comptes rendus accordés antérieurement (entre 1756 et 1759) à l'Encyclopédie, du fait de son interdiction et de celle du Journal lui-même, alors à Liège, par les Théologiens de Louvain. II aura eu le temps, avant l'obligation de fuir Liège pour Bouillon, d'analyser des articles du volume 5 sur un nombre de pages tellement étendu que l'espace réservé à l'Encyclopédie prit une ampleur démesurée : calculée à partir de l'indice 100 des comptes rendus accordés au Dictionnaire, la quantité des pages consacrées aux autres sujets de l'actualité éditoriale affichent un indice trois fois moindre (27). Du fait de cette (trop) grande fidélité à l'Encyclopédie, le Journal encyclopédique perdait son profil agréable et diversifié, si caractéristique des périodiques du siècle, pour devenir le journal savant de l'Encyclopédie ${ }^{14}$. C'est donc à juste titre qu'il se glorifia dans une sorte de récapitulatif de son travail de recension, rédigé en 1770, d'avoir été aussi fidèle que possible au grand ouvrage :

Depuis environ quatre années que nous avons repris la suite de ce Dictionnaire, nous en avons fait connaître, autant que notre zèle et nos lumières nous l'ont permis, sinon tous les articles les plus savants, du moins les plus intéressants soit pour les gens de lettres, soit pour les personnes qui ne cherchent qu'à s'éclairer sur les matières relatives à l'administration publique, ou sur les différents objets d'économie particulière (JE, 15 juillet 1770, p. 168).

Et de fait, hors les années qui suivirent l'interdiction et de l'Encyclopédie et du Journal en 1759, qui imposa un silence durable au journaliste (six années entières), les comptes rendus des articles de l'Encyclopédie se suivirent régulièrement et presque toujours en

condition de disciples à celle d'amateurs et de celle-ci à la profession de philosophes » (De l'interprétation de la nature, XXXIX, éd. Vernière, Garnier, 1961, p. 214).

12 En 1771, il donnera place à une étude de Dutens consacrée aux « différents éditions de l'Encyclopédie », transmise par le libraire Gosse de La Haye ; il lui associa « quelques notes essentielles » venues des éditeurs suisses de la nouvelle version de l'Encyclopédie, qui rectifient des erreurs du destinateur (Gosse) plus favorable à l'édition d'Yverdon (de Felice) qu'à l'édition de Paris (JE, IV, p. 445-454)

${ }^{13}$ Voir sur ces questions, notre thèse Lecture et société dans le Journal Encyclopédique de P. Rousseau (1756-1785), Clermont-Ferrand, 1987, vol.1, p.236 et sq.

14 Ibid, p.242. II faudrait aussi intégrer à ce calcul, les multiples occasions que se donne le journaliste pour renvoyer à l'Encyclopédie dès qu'un mot la lui rappelle comme ce fut le cas à propos d'un titre de Velat contenant une section intitulée Goût (Poliergie, Duchêne, 1756) II signale le futur résultat « précieux » obtenu par Diderot sur le canevas de Montesquieu (JE,15 mars 1757, p. 7). 
première place dans chaque numéro sur environ 20 à 30 pages. Seuls quelques événements éditoriaux exceptionnels les reléguèrent à un autre numéro, en usurpant la première place : les CEuvres de Montesquieu ${ }^{15}$; De l'Esprit d'Helvétius plus tard ${ }^{16}$. Des thèmes encyclopédiques seront représentés, en l'absence du Dictionnaire, durant l'année 1759, par des ouvrages sur le commerce (chevalier d'Arcq), sur les finances de France (Véron de Forbonnais), sur le Beau (P. André), sur les sentiments moraux (A. Smith), sur les Mathématiques (Principes mathématiques de la philosophie naturelle de Mme Du Châtelet ; ou les Opuscules mathématiques de d'Alembert), sur le droit naturel (Hubner, puis Wolff), sur le goût (Gérard), sur la poétique (Marmontel), sur la philosophie de Leibniz, comme si, aux yeux de P. Rousseau, l'esprit encyclopédiste imprégnait toute la production éditoriale de ces années. Certains ouvrages permettaient de dépasser les livres du temps présent et de rédiger des comptes rendus dignes d'un article du Dictionnaire $^{17}$ : ainsi de l'Homme de lettres de J.-J. Garnier, de la présentation des Philosophes modernes par Savérien ou les Vues philosophiques de Prémontval, etc. Même pendant l'interdiction de l'Encyclopédie, le Journal restait largement proche d'elle par le choix philosophique de ses principales recensions, opérées attentivement par P. Rousseau. Il avait mis en œuvre une méthode pour éviter l'échec de ses promesses, inspirée de Diderot évoquant le risque de réduire son grand ouvrage à « une compilation très informe et très incomplète » (Enc., vol.V, art. "Encyclopédie », p.645a). II a dû éprouver un immense sentiment de soulagement en lisant Diderot mettant fin à une série morose de repentirs tardifs : "Mais, dira-t-on, comment avec tous ces défauts vous est-il arrivé d'obtenir le succès qu'aucune production aussi considérable n'a jamais eu ? C'est la suite de l'heureux choix de ceux qui s'y sont consacrés » (ibid.). II s'appuya à son tour sur ce principe du choix ${ }^{18}$ pour répondre à ses exigences et transformer son journal en ouvrage 'encyclopédique', grâce à l'« enchaînement des connaissances ${ }^{19}$, obtenu parfois par l'ordre qu'il s'efforçait de mettre dans la succession de ses comptes rendus ou dans les comptes rendus eux-mêmes ; mais surtout, par un usage réfléchi de l'esprit encyclopédique, probablement moins ordonné mais du moins aussi « raisonné » que le Dictionnaire dont l'exemple le fascine et l'inspire ${ }^{20}$, en dépit des différences multiples des deux genres littéraires. Sous le regard impressionnant et fiévreux de son aînée ${ }^{21}$, il tenta de mettre en œuvre deux types de réponses pour placer son journal à la hauteur de ses maîtres : l'un rhétorique (l'ordre encyclopédique), l'autre moral (l'esprit philosophique). $P$. Rousseau insiste sur cet aspect rhétorique de son travail dès le premier numéro de janvier 1756 par souci déontologique du confort pédagogique de ses lecteurs : « Nous nous attacherons à mettre un ordre si simple et si naturel qu'on pourra jouir du fruit de notre travail sans y prendre la moindre part » (JE,1756, Avertissement, p. XIV). II imagina naïvement qu'il trouverait une réponse à ce problème de masse et de désordre propre aux compilations journalistiques dans une technique de mise en relation d'ouvrages faciles à rapprocher : «Il est essentiel de différer quelquefois un extrait d'un ou deux mois afin de

\footnotetext{
15 JE, 15 août 1758, p. 3-26 ; 1er septembre 1758, p. 3-26 ; 15 septembre 1758, p. 3-25.

$16 \mathrm{JE}, 1$ er octobre, 15 octobre et 1er novembre 1758.

17 P. Rousseau a été séduit par l'article «Droit naturel » de Boucher d'Argis, qui répond à ses propres conceptions.

${ }^{18}$ Ce principe s'opposait frontalement aux devoirs de présentation des nouveautés attachés au métier de journaliste; Diderot l'a indiqué à dessin comme solution à la médiocrité des entreprises culturelles, encyclopédies ou journaux, car il révélait la qualité de la compétence intellectuelle du rédacteur.

${ }^{19}$ Diderot, art. « Encyclopédie », in Enc., vol.V (1755), p.635b.

${ }^{20} \mathrm{P}$. Rousseau est capable de séparer le désordre propre à Diderot et son génie, comme à propos de son article 'Encyclopédie' : « L'ordre n'y est pas exactement suivi; mais il n'est interrompu que par des écarts de génie bien supérieurs aux proportions géométriques » (JE, février 1756, p.16).

${ }^{21} \mathrm{P}$. Rousseau était né en 1716 , trois ans après Diderot.
} 
le rapprocher d'un autre qui traitera le même sujet et qui sera le développement du premier. Cette suite d'idées que nous entretiendrons autant qu'il sera possible établit un ordre encyclopédique » (JE, 1er janvier 1757, p. 92).

Dans ces perspectives diderotiennes, il lui arrive de rompre le pacte journalistique imposant de suivre fidèlement l'actualité. À l'occasion de la parution du second volume du Recueil des Antiquités de Caylus, il n'hésite pas à revenir en arrière : « Nous avons trop à cœur ce précieux enchaînement des Arts et des Sciences que le titre de notre Journal présente, pour ne pas rapporter ici le ler volume » (1er mars 1756, p. 62-63 (II-2). Pour répondre par avance à un agacement probable de ses lecteurs, il souligne qu'il ne fait que respecter fidèlement ses engagements initiaux, plus exigeants que ceux des autres journalistes :

Les journalistes qui l'ont annoncé, ne sont entrés dans aucun détail. Ce trait de prudence aurait dû nous servir d'exemple ; mais nous connaissons nos engagements, nous les avons sans cesse devant les yeux, et si jusqu'à présent nous avons la douleur de ne les avoir pas emplis, du moins s'appercevra-t-on [sic] du désir que nous avons de le faire (ibid., p. 63).

Son respect légitime de ses lecteurs ne le distrait pas de son respect prioritaire de l'ordre encyclopédique, emblématique de la raison en acte. Son souci d'ordre se manifeste aussi et plus encore dans le traitement qu'il réserve aux articles de l'Encyclopédie. II en bouscule volontiers et fréquemment l'ordre de succession alphabétique. II opéra de cette manière à plusieurs occasions. La plus significative concerne l'article « Encyclopédie », le premier du Volume $V$ qu'il retient dans son deuxième tome en février 1756, pour s'engager dans « l'immense extrait » du Dictionnaire qu'il prévoit de mener à son terme. Cet article est analysé avant ses nombreux prédécesseurs alphabétiques ${ }^{22}$. P. Rousseau s'en explique en recourant à l'argument de « l'enchaînement des connaissances » qui résume le mot et l'acte encyclopédiques. La logique de son travail, inspirée par Diderot, lui importe plus qu'une fidélité mécanique et formelle à la réalité bibliographique. II veut traiter, en effet, sa matière première en encyclopédiste soucieux d'établir des « rapports » entre des réalités dispersées et séparées, selon l'un des mots et l'une des idées préférés de Diderot. À défaut d'être autorisé par le genre périodique à rédiger des comptes rendus aussi complets qu'un article de l'Encyclopédie, il transforma leur succession éparpillée en étapes coordonnées par la fin poursuivie : l'établissement d'un tableau des savoirs modernes présenté sous la forme antagonique d'un cheminement « raisonné ». Le temps s'inversait en atout. C'est pourquoi, il prépara soigneusement et systématiquement, dans les deux premiers numéros du journal en janvier 1756, la future livraison de ses comptes rendus consacrés à l'Encyclopédie, effectuée seulement à partir du 15 février. En effet, il débuta son travail de recension par un ouvrage anglais dont il n'apprécie pourtant pas la facture « frappée au coin de la littérature anglaise ». La référence nationale n'a rien de flatteur:

Les épisodes fatiguent, c'est moins l'histoire de Bacon que de l'Angleterre...moins parfaite, moins agréable, elle est d'autant moins la vie de Bacon ; le style en général en est négligé ${ }^{23}$.

\footnotetext{
22 II ne sera accompagné que par ceux de d'Alembert sur les Élémens des sciences et de Boyer d'Argis sur le Droit naturel (et non celui de Diderot) et sur la Dot.

${ }^{23}$ Le rédacteur ne parle-t-il pas ici de son propre style sans en prendre conscience ? Car, écrivant vite, il ne s'écoute pas (/enéné/).
} 
Cet ouvrage dont l'auteur et le traducteur « ne se réveillent que » trop rarement, lui est pourtant utile, dans l'attente de celui de Deleyre ${ }^{24}$, car il contient un exposé de Bacon sur le Rétablissement des sciences qui présente, aux yeux du journaliste, une première version de « la Théorie universelle des Arts et des Sciences » (JE,1er janvier 1756, p. 11). Cet exposé baconien, de belle tenue et de grande ampleur, dont le journaliste résume consciencieusement les six parties, le conduit à annoncer son intérêt pour l'Encyclopédie, dans la mesure où " on sait combien les auteurs du Dictionnaire Encyclopédique ont perfectionné ce système, sans diminuer la gloire de Bacon, qui en est le Créateur » (ibid., p. 12). La mort n'a pas octroyé au philosophe anglais le temps suffisant pour aboutir au « terme de ses travaux » et pour en faire « l'objet le plus sublime »; la " chaîne philosophique d'axiomes qu'il eût pu intituler Éléments de la Philosophie » ${ }^{25}$, sera écrite, lance P. Rousseau, par les deux responsables de l'Encyclopédie comme héritiers directs de Bacon :

Cet ouvrage devait être le terme de ses travaux. II le laisse comme une succession précieuse aux Génies qui viendraient après lui. Cette espérance fut la consolation de ses derniers jours, et fait aujourd'hui la gloire de notre siècle (ibid., p. 15).

Un premier jalon est ainsi posé, qui sera complété par la lecture des Pensées de l'interprétation de la nature qui confirme Diderot dans ce rôle privilégié de successeur héritier de Bacon. Après bien des modifications imposées par $P$. Rousseau à cette œuvre ${ }^{26}$, le compte rendu se clôt sur la célébration de «l'union des gens à talents » illustrée par « cette ligue philosophique si glorieuse de l'Encyclopédie » (JE, 1er janvier 1756, p. 16). Le chemin conduisant à l'Encyclopédie est donc bien balisé et l'insertion du premier article extrait du premier volume, justiciable d'un compte rendu au vu de sa date de parution (vol. 5 de 1755), juste avant le lancement du premier numéro du journal, n'est plus liée à un simple exercice d'information : elle est rattachée consubstantiellement à l'ordre encyclopédique de l'enchaînement. Du tout premier compte rendu paru dans le journal au premier compte rendu portant sur le grand Dictionnaire, il n'y a pas de solution de continuité. Le choix, effectué par $P$. Rousseau, des ouvrages à recenser, dessine un itinéraire raisonné qui, échappant aux amas désordonnés d'une bibliothèque purement cumulative, aboutit à l'imposition d'un ordre et d'une direction. Débarrassé de l'écume des jours tant appréciée par les amateurs de périodiques, il mène au saint Graal encyclopédique : «Le plus grand éloge qu'on puisse faire aujourd'hui du Chancelier d'Angleterre, c'est de donner ici l'Encyclopédie comme une suite de ses ouvrages et comme une émanation de son génie » (JE,15 février 1756, p. 3). Ce geste constitue le premier maillon de la chaîne construite d'un numéro à l'autre du journal dans les trois premiers mois de 1756.

Le second sera, comme annoncé plus haut, l'article « Encyclopédie » inséré, grâce à un déplacement contrevenant à l'ordonnance matérielle du Dictionnaire. P. Rousseau prépare son lecteur en lui assurant que cet article est à lire d'abord car « il contient la discussion du plan et des matériaux de tout l'Ouvrage, et un projet raisonné pour ceux qui auraient le courage d'en entreprendre un meilleur » (JE, 15 février 1756, p. 5). Cette manière inhabituelle relève de l'exceptionnelle ampleur de la réflexion : "L'objet principal y est traité sous toutes ses faces. Ce qu'il y a d'admirable, ce sont les grandes vues qui y sont jetées à profusion... » (ibid., p. 16.). Elle réélue aussi d'un souci rhétorique. Cet

\footnotetext{
${ }^{24}$ Deleyre (Alexandre) : Analyse de la philosophie du chancelier Bacon (1er février 1756, p. 3-19).

${ }^{25}$ Le rédacteur de ce compte rendu s'inspire bien évidemment de l'article de d'Alembert, lu à la sortie du Volume V, en 1755 : Éléments des sciences, qu'il examinera à la suite de l'entrée Encyclopédie le 15 février 1756.

${ }^{26}$ II n'était pas envisageable de les analyser dans les bornes de cet article.
} 
article, lui paraissant éclairer toute la démarche, la méthode, l'histoire du grand ouvrage jusqu'à en détailler avec franchise les insuffisances et les imperfections, exigeait, pour guider au mieux les lecteurs dans son immensité, une présentation préalable dont il lui revenait le devoir de la rendre accessible à tous malgré sa taille : « Un lecteur un peu paresseux sera effrayé de l'étendue de l'article Encyclopédie »; or, précise le journaliste, « il est dans l'ordre de proportion ». (JE 15 février 1756, p.5). Cette dimension matérielle tellement hors norme ${ }^{27}$ est (à) l'image de la dimension intellectuelle du Dictionnaire, comme si la quantité de matière du corps typographique symbolisait la qualité spirituelle d'une âme en expansion. La fidélité à l'esprit de l'ouvrage commandait ce bouleversement inédit de l'ordre des matières retenu par le journaliste ; il se glorifie d'être un éclaireur dévoué au service, non seulement de la philosophie en acte qu'est ce Dictionnaire et des grands hommes qui en sont les auteurs, mais au service du public engagé dans une démarche philosophique de conquête du savoir : "Nous souhaitons que le Public prenne à la lecture de cet extrait, le même plaisir que nous avons pris à le faire » (JE, 15 février 1756, p.15) ${ }^{28}$. Le journaliste effectue donc une lecture active du Dictionnaire, évitant de se reposer passivement sur l'organisation originelle du document : il en abandonne la présentation alphabétique pour opérer sa sélection sur un mode « raisonné » qui correspond à un choix hiérarchisé de type à la fois intellectuel et rhétorique ${ }^{29}$. Guidé et soutenu par l'enthousiasme de Diderot, il finit par découvrir une réponse à la question de sa légitimité qui le préoccupe $\operatorname{tant}^{30}$ : un périodique bien pensé peut se mettre, toute proportion gardée, à la hauteur d'une entreprise encyclopédique, ne serait-ce qu'en empruntant une voie philosophique que le genre n'interdit pas ; il lance un défi à Diderot :

Nous demanderons à ceux qui en ont une si petite idée, si ce n'est pas plutôt la faute des auteurs que du genre lui-même...Pourquoi ce genre, s'il était manié par ses mains habiles, ne pourrait-il pas être annobli [sic] et s'élever jusqu'à devenir un journal philosophique, et par cela même contribuer au développement de l'esprit humain? » (JE,15 novembre 1756, p. 7).

L'élévation indispensable indique la conscience persistante, en P. Rousseau, de la petitesse, pour ne pas dire médiocrité, si couramment attribuée à l'univers journalistique ; il répétera, un an plus tard, ce terme au moment d'établir un premier bilan de son travail : à la vue d'une «époque si riche » de nouveaux savoirs et de génies créateurs, « il faudrait qu'un journaliste eût un génie bien stérile s'il ne rendait pas son journal intéressant » à la condition qu'il acceptât de « s'élever avec son siècle », en obéissant, non plus « aux autorités, mais aux lois de la raison » (JE, 15 nov. 1757, p. 14 et 16). II reprenait une argumentation extraite de l'article « Encyclopédie » : «Si le gouvernement se mêle d'un pareil ouvrage, il ne se fera pas... Une Encyclopédie ne s'ordonne point » (Enc., vol. V,

\footnotetext{
${ }^{27}$ Le journaliste précisera, à la fin de son analyse de cet article, à la fois pour en marquer l'énormité et indiquer son mérite de journaliste dévoué et philosophique, qu'il s'étendait sur « 200 pages in $-12^{\circ} \gg$.

${ }^{28}$ Nous donnons cet exemple de modification de l'ordre externe de la réalité bibliographique au profit d'un ordre interne inspiré à $P$. Rousseau par imitation du comportement encyclopédique, car il est emblématique d'une manière d'opérer familière au journaliste.

${ }_{29}$ II eût convenu de citer ici le cas, entre bien d'autres, de Femme (de Démahis), préféré à celui de Boyer d'Argis, placé avant « Fat » utilisé en commentaire moqueur à l'encontre de son auteur (JE, 15 octobre 1756, p. 59-79, à la réputation, partagée avec Cahuzac, de « petits freluquets » dont « les puériles déclamations déshonorent un si bel ouvrage " selon Voltaire (Corr. générale, éd. Best., Paris, Gallimard, t. VII, lettre. 8503, p. 856); mais il enchantera P. Rousseau pour « les jolis portraits et les détails charmants » de sa comédie L'Impertinent quelques semaines plus tard (JE, 15 déc. 1756, p. 124).

${ }^{30}$ Nous laissons de côté, dans cadre de cet article, la troisième voie de légitimation suivie par $\mathrm{P}$. Rousseau : le partage des malheurs.
} 
p. 636a). II s'accorde avec Diderot sur le niveau culturel des esprits de son temps qu'il est indispensable d'améliorer pour comprendre l'esprit encyclopédique d'un Bacon : « Les esprits ne sont pas assez avancés. II y a trop peu de personnes en état de s'élever à la hauteur de ses méditations, et peut-être le nombre n'en deviendra-t-il jamais guère plus grand » (p. 647a) ». À ses yeux, l'élévation doit, pour réussir, respecter l'esprit des lecteurs, comme le rappelait Diderot de son côté : « Un éditeur qui aura de l'expérience, et qui sera maître de lui-même, se placera dans la classe moyenne des esprits « (ibid., p.648b). En modifiant ainsi profondément la conception du métier de journaliste par ses références aux orientations de Diderot et son désir d'être l'un de ses thuriféraires autorisés, $\mathrm{P}$. Rousseau réussit à s'introduire dans le champ encyclopédique sans perdre de vue les contraintes de son activité périodique. La réponse était donc ainsi donnée aux inquiétudes qui tourmentaient, très pertinemment, le journaliste : comme Diderot, il réussit à associer le respect du travail philosophique de formation des esprits, (la révélationdivulgation des savoirs) aux exigences élevées de la raison (le renouvellement des sciences). II s'efforçait de transformer l'actualité en modernité au sens où Diderot avait, à l'indignation de certains ${ }^{31}$, déclaré :

Aujourd'hui que la Philosophie s'avance à grands pas; qu'elle soumet à son empire tous les objets de son ressort ; que son ton est le ton dominant, et qu'on commence à secouer le joug de l'autorité et de l'exemple pour s'en tenir aux lois de la raison, il n'y a presque pas un ouvrage élémentaire et dogmatique dont on soit entièrement satisfait... Le temps est arrivé, où des ouvrages, qui jouissent encore de la plus haute réputation, en perdront une partie, ou même tomberont complètement dans l'oubli... Si l'on en excepte ce Perrault et quelques autres, LaMothe, Terrasson, Boindin, Fontenelle..., il n'y avait peut-être pas un homme qui en eût écrit une page qu'on daignât lire aujourd'hui » (loc.cit., p.636b).

La modernité coïncide avec l'obsolescence des pensées anciennes. Le journaliste s'est donc voulu moderne ${ }^{32}$ dans le sillage de Diderot mais sans accepter ses excès.

P. Rousseau aura beaucoup travaillé à effacer le fossé que Diderot avait creusé entre périodique et Encyclopédie, tout en rejetant certains conseils inadaptés d'un philosophe tout à sa passion militante : « combien ces ouvrages périodiques seraient abrégés, si on laissait seulement un an d'intervalle entre la publication d'un livre et le compte rendu qu'on en rendrait: l'ouvrage dont on a parlé fort au long dans le journal, n'y serait pas même nommé » (art. «Encyclopédie », vol.V,1755, p.645a). II lui fallut bien de l'énergie et de la persévérance pour contredire les jugements abrupts et les conseils impraticables de ce maître tant vénéré. Il en fit son Surmoi sans daigner en faire un Idéal du Moi, car il eut vite conscience des différences des rôles socio-culturels que chacun dans son champ particulier avait à jouer tout en partageant les mêmes finalités : à l'écrivain de génie de heurter les habitudes sans tergiverser, au journaliste à s'adapter, sans s'y conformer, aux capacités du public. En ce sens, P. Rousseau, bien qu'il manifestât une fidélité continue au Diderot philosophe (plutôt qu'au Diderot écrivain) fut

\footnotetext{
${ }^{31}$ Un lecteur de l'Année littéraire, déclarant avoir quitté Paris pour habiter la campagne (signe de bon sens!) depuis quinze ans, s'étrangla d'indignation à la lecture de ces lignes qu'il cite : " Je vois évidemment que les idées que j'ai eues jusqu'à présent sont d'une absurdité à me faire passer comme un imbécille [sic], un Ostrogoth...À mes yeux paraît la statue de Diderot en beau marbre de Paros, placée au fond du sanctuaire, tenant ainsi que l'illustre évêque d'Hippone, un grand livre ouvert d'une main, une plume d'or de l'autre et tous les peuples à genoux, qui l'adorent comme l'organe de la raison, l'oracle de la vérité, l'interprète de la nature, le bienfaiteur du genre humain » (A.L. III-lettre11, p.245 et 248).

${ }^{32}$ Voir sur ce point notre article sur La Modernité dans le Journal Encyclopédique (1756-1786), RHLF, 42002, p. 545-561.
} 
plus voltairien que diderotien ${ }^{33}$; car il n'était pas en mesure d'approuver les audaces et les impertinences « décomplexées » de ce maître encyclopédiste, bien trop libre pour accepter et supporter les retenues ou les timidités de la décence propre à l'honnête homme classique ${ }^{34}$. Insérer des articles du Dictionnaire et lire les textes de Diderot transformèrent le petit auteur d'agréables comédies divertissantes ${ }^{35}$ en journaliste philosophe à la manière encyclopédiste définie par Diderot. Au point que très tôt, il se sentit capable de remplacer la première équipe encyclopédique et de poursuive à sa manière le travail de Diderot:

S'il est heureux qu'une Société savante s'applique sans relâche à recueillir tout ce qu'il y a d'essentiel dans les connaissances humaines et si très peu de gens peuvent acquérir cet immense recueil, il est heureux qu'une autre société s'applique à faire un choix nouveau, et qu'après avoir soumis les meilleurs morceaux aux lois d'une discussion exacte, elle en présente la substance à tous ceux qui méritent d'en jouir, et qui savent les apprécier (JE, 15 oct.1756, p.60).

De la sorte, ce périodique deviendrait le meilleur agent littéraire, en Europe, du nouveau monde des Lumières : "Aucun Journaliste de l'Europe n'a songé à procurer cet avantage à ses lecteurs ; aussi n'ont-ils pas le bonheur de compter parmi leurs souscripteurs autant de philosophes que nous ; nous avons le rare plaisir de converser avec nos maîtres » (Ibid.). P. Rousseau ne semble-t-il pas, dès ce premier numéro, pasticher le projet de Diderot et d'Alembert, en présentant son journal comme une encyclopédie-bis ? Malgré les pénibles inconvénients d'un tel engagement, comme il le révèlera dans son dernier compte rendu de $1770^{36}$ :

Quelque soin que nous ayons pris pour ne rien omettre de ce que nous avons jugé pouvoir rendre nos extraits plus instructifs, et quelques précautions que nous ayons employées pour ménager la sensibilité des auteurs, nous nous sommes vus quelquefois obligés de critiquer les défauts ou de combattre les erreurs ; nous n'avons pourtant point satisfait tous nos lecteurs et il s'en faut de beaucoup que les auteurs des articles que nous avons successivement rapportés, aient été contents de nos observations que nous avons cru nous permettre » (JE , 15 juillet 1770, p. 168).

Encyclopédiste, oui, jusqu'au martyre inclus, oui ; tel fut le sort de P. Rousseau recevant Diderot dans le salon de ses pages, durant près de vingt années, métamorphosé par une

\footnotetext{
${ }^{33}$ Comme Voltaire le fit remarquer dans sa première Lettre anglaise : « on ne se défait pas de ses habitudes tout d'un coup » (Lettres philosophiques, Bâle, 1734, p. 2).

${ }^{34}$ Saint-Lambert, cité par P. Rousseau (JE, avril 1767, p. 2-19), rappellera, dans l'article « Honnête » de l'Encyclopédie la nécessité de maintenir fermement les passions dans des limites raisonnables, probablement contre les idées de J.-J. Rousseau et peut-être de Diderot, que Palissot avait visé en 1757, à la suite de Fréron se moquant des premiers mots de l'Interprétation de la nature : «On vit à la tête de quelques productions philosophiques un ton d'autorité et de décision qui, jusqu'à présent, n'avait appartenu qu'à la chaire. On transporta à des traités de morale, ou à des spéculations métaphysiques, un langage que l'on eût condamné, partout ailleurs comme celui du fanatisme » (Petite lettre sur de grands philosophes, p. 3, (Paris, IV-101, in-12). Palissot cite les Pensées philosophiques, le «livre obscur » des Pensées sur I'Interprétation de la nature et le Discours sur l'inégalité des conditions.

${ }^{35}$ II composa Les Méprises, L'Esprit du jour, Le berceau, Les Faux pas, lues et appréciées par Fréron.

${ }^{36}$ Sur les premiers suppléments : Affabilité, Bibliotaphe, Enthousiasme, Glorieux, remarquons que l'ordre alphabétique est préservé mais que le rédacteur utilise, à son propre usage, l'entrée 'Glorieux' perçue comme une « excellent esquisse d'un caractère qui n'est que trop commun dans les sociétés de toutes les espèces, sans même en excepter les plus éclairées »; il joue, en conclusion de son dernier compte rendu de l'Encyclopédie, au moraliste classique à la manière de La Bruyère pour rire de la comédie humaine.
} 
innutrition consciente à la Du Bellay ${ }^{37}$ mais sans regrets ${ }^{38}$, exemple inattendu d'une « relation existentielle à la littérature ${ }^{39}$.

Jacques Wagner. UMR IHRIM-5317.

${ }^{37}$ Sonnet XXXII des Regrets : « Je me ferai savant en la philosophie » (1ère éd., Paris 1558), p.22.

${ }^{38}$ Gide parlerait peut-être de « cette intime connaissance qui est comme le sentiment d'une parenté retrouvée » (Essais critiques, cité dans «Gide historien de la littérature » par Marielle Macé (Bulletin des Amis d'A. Gide, XXXV,153, 2007, p.78-79).

39 «La vie se donne à lire comme tout entière dirigée vers la constitution de l'œuvre, et c'est cette tension, cette insistance, cette permanence qui est une réussite « (Barthes, cité par M. Macé, Ibid.). Certains, plus réalistes, parleraient de concurrence économique entre éditeurs. 